\title{
Mobile Motorcycle Lift for the Common Man
}

\author{
Joseph Timothy Foley ${ }^{1, \star}$, Guðrmundur Pórir Sigurðsson ${ }^{1}$ Jóhann Smári Gunnarsson ${ }^{1}$, Jón Gautason ${ }^{1}$, and Ólafur \\ Jósef Ólafsson ${ }^{1}$ \\ ${ }^{1}$ Reykjavik University, Menntavegur 1, Reykjavik 101, Iceland
}

\begin{abstract}
Motorcycle enthusiasts often need to spend significant amounts of time tuning and improving their bikes. Traditionally this is done by hobbyists by using the bike's own stand which can be dangerous due to its instability. For those with a little more money, a dedicated free-standing bike stand is the fixture of choice. Unfortunately, many of the bike stands are too expensive, too big, or have very weak ergonomics. In this paper, we present a motorcycle lift designed using Axiomatic Design that has a small footprint, is adjustable for a large range of different bikes and users, and can be mounted without the user lifting the bike. Though this prototype design is more expensive than the simple bike stands, we believe its functionality makes it well worth the extra cost.
\end{abstract}

\section{Introduction}

The goal of this project was to design a motorcycle lift focused on the practical maintenance needs of a motorcycle owner that are lacking in affordable lifts. The most common complaint is that the bike can't be moved around when you have removed the wheels. This is often discovered when using the most common low-cost lifts that are commonly employed: milk crates and other milk storage devices. Amateur motorcyclists often cannot afford a space devoted to just one ongoing project, so there is a need for mobility while minimizing the footprint of the lift. In addition, the lift should take up very little space when not in use to free up space for other projects in your garage or other work-spaces.

Like the name implies, the Mobile Motorcycle Lift is designed to lift up a motorcycle both to do maintenance on it and for safety inspections before each ride. Every motorcycle rider that rides on dirt bikes or road bikes needs a lift with these features to perform safety inspections and regular maintenance. Motorcycle owners dream of having a fully-featured motorcycle lift as found at the professional mechanic, but such lifts are far out of the financial means of most owners. Our goal was to make the perfect motorcycle lift as a careful balance of features and cost.

\section{Prior art}

The majority of low-cost lifts use a scissor configuration to convert an actuator that is very high force into sufficient displacement while keeping the entire mechanism compact. A motorcycle is positioned such that the engine or the wheel engages a locking device; often a retaining strap

\footnotetext{
^e-mail: foley@ru.is
}

is used to fixture the bike to the top surface. Once the motorcycle is secured, either a crank is turned or a hydraulic mechanism is engaged.

Our survey of existing lifts showed that some of the common complaints from our user base were not addressed by the existing lifts. People wanted to a way to change the oil quicker and without spilling it all over the garage. Others were irritated that there was never enough space to put down their tools. All wanted a lift that was mobile so that different areas were easier to access and the bike could be put temporarily in storage while it was being worked on.

There is also one design flaw found in many of the lifts: when the front of the motorcycle is being taken apart, the lifts may tilt back uncontrollably until the back wheel of the motorcycle hits the ground. Many lifts take up too much space when not being used as well as not lifting high enough.

On the lower price range of the lifts, a common complaint was that the motorcycle itself had to be lifted onto the mechanism. When dealing with the heavy load of a motorcycle, this can easily translate into pulled muscles and a strained back. Everyone surveyed was excited for a lift that the motorcycle could be rolled onto or could slide underneath the bike.

The Black Widow lift (Figure 1) is small and compact and mobile. Unfortunately, the wheels can't easily be disengaged while in use since the wheels are attached to a separate dolly (Figure 1-c) that can be put under the lift [1]. Disengaging the wheels would require the user to remove the dolly from under the lift while the bike is on top: this would require lifting the entire weight of the motorcycle and the lift. The lift can also be used without the wheels but then it can't be moved around: Figure 1-b shows the lift configuration without wheels. This design only has a 


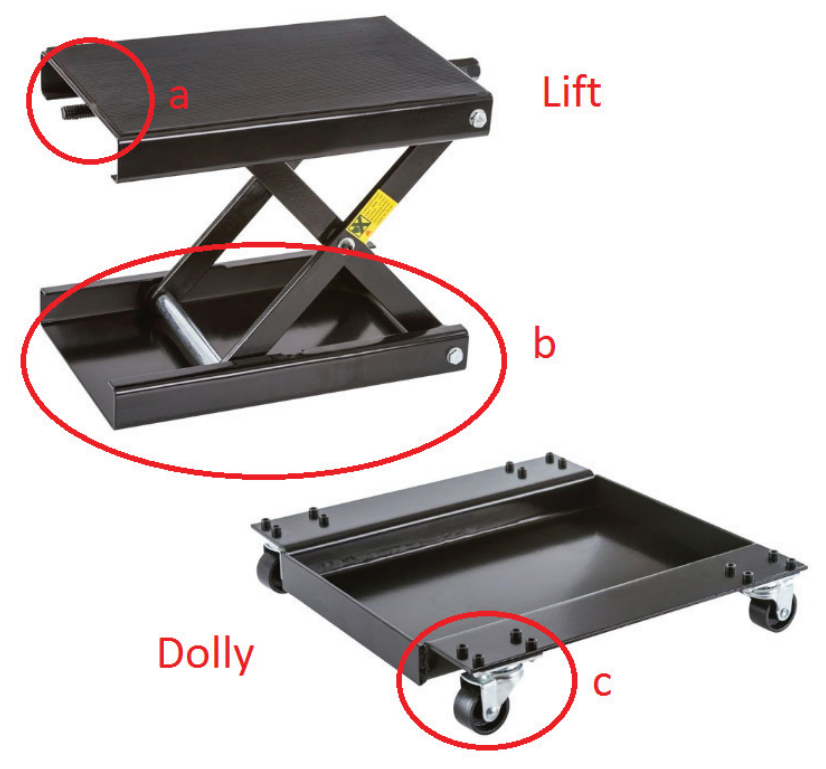

Fig. 1. Black widow lift [1]

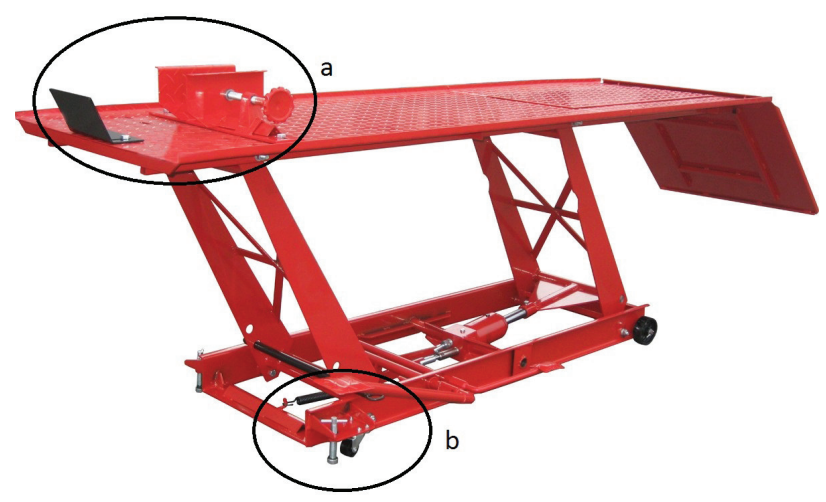

Fig. 2. Pennsylvania Motorcycles lift [2]

manual screw jack system, not a hydraulic jack (Figure 1a). Without a hydraulic jack, the lifting process is a lot slower and takes more effort to lift. In addition, no provision is made in this jack design for securing the bike to the lift.

A lift from Harbor Freight made by Pittsburgh Motorcycle is shown in Figure 2. This device lifts the motorcycle to a good work height using a hydraulic piston and has wheels that can be disengaged before the motorcycle is put on top (see Figure 2-b) [2]. The motorcycle is rolled up the ramp and screw-clamp is tightened to hold the front wheel. This process is slow for securing the wheels making it not convenient if the lift is often moved around. However, the main drawback of this design is the fact that it takes up a lot of space while it's not being used. There are also concerns that the clamp on the front wheel (Figure 2a) is not secure, resulting in an unstable mount. Even if the clamp is sufficient, this lift is incompatible with work being performed with the front wheel removed. The large footprint of the lift provides good stability but at a cost of large weight and inconvenient storage.

\section{Design}

We began the process of designing our "perfect" amateur motorcycle lift based upon a survey of complaints from motorcycle enthusiasts we knew in Iceland. We applied the Axiomatic Design methodology to structure and focus the complaints into a coherent set of needs (Section 3.2) from which requirements (Section 3.3) could be drawn up.

\subsection{Design Methodology}

Axiomatic design provides the theoretical foundation to generate and evaluate design solutions of complex systems [3]. It is a systems design methodology using matrix methods to systematically analyze the transformation of customer needs into functional requirements, design parameters, and process variables. Axiomatic Design as a mechanical design process focuses on the definitions of Functional Requirements (FRs), Design Parameters (DPs) and their interrelationship through a process of mapping between the functional domain and the physical domain.

The design process is to select the best set of DPs to satisfy the given FRs following certain criteria [4]. One goal of Axiomatic Design is to minimize the number of DPs, which can achieve FRs independently [3]. Specifically, functional requirements (FRs) are affected to design parameters (DPs) in a manner of "coupling". FRs and DPs coupling is communicated in a design matrix as shown in Equation 1. In this design matrix, coupling is represented as non-zero coefficients.

There are two axioms used in this method: Axiom 1 states that the independence of the FRs must be maintained, and Axiom 2 states that the information content should be kept at minimum [3]. In short, designers must minimize the coupling on non-diagonal elements of the design matrix and choose implementations (DPs) that meet their coupled requirements with the highest probability. After analyzing the customer's needs, we were able to set functional requirements and then design parameters. An example of an "uncoupled" Design matrix can be seen in Equation 1. For two requirements (FRs) and two implementations of those requirements (DPs), this is considered an optimal design with respect to coupling (Axiom 1).

$$
\left[\begin{array}{l}
\mathrm{FR}_{1} \\
\mathrm{FR}_{2}
\end{array}\right]=\left[\begin{array}{cc}
\mathrm{A}_{11} & 0 \\
0 & \mathrm{~A}_{22}
\end{array}\right]\left\{\begin{array}{l}
\mathrm{DP}_{1} \\
\mathrm{DP}_{2}
\end{array}\right\}
$$

\subsection{Customer needs}

The focus group (customer) for this product would be regular motorcycle owners, particularly off-road riders who 
have to do a lot of maintenance to their bike, e.g. tire and oil change, suspension, and clutch work. The goal of any motorcycle lift is to make maintenance on the motorcycle easier. This top-level goal is described as $\mathrm{CN}_{0}$. From this top-level goal, the rest of the needs are decomposed.

- $\mathrm{CN}_{0}$ : Better motorcycle maintenance working conditions

$-\mathrm{CN}_{1}$ : Easy storage

- $\mathrm{CN}_{2}$ : Compact when stored

$-\mathrm{CN}_{3}$ : Motorcycle secure and stable on lift to prevent any possible danger due to it falling.

- $\mathrm{CN}_{4}$ : Fit under the motorcycle so it doesn't require being lifted on to the lift

$-\mathrm{CN}_{5}$ : Can be moved around

\subsection{Functional requirements}

Functional requirements (FRs) are a minimum set of independent requirements that completely characterize the functional needs of the design solution in the functional domain. [3] The functional requirements below are decomposed from a top-level $\mathrm{FR}_{0}$. As per best practices [4], each FR must start with an action or transformational verb and be verifiable.

- $\mathrm{FR}_{0}$ : Make maintenance easier than with existing lifts for the average person.

- $\mathrm{FR}_{1}$ : Lift motorcycle to comfortable working height.

- $\mathrm{FR}_{2}$ : Keep work-space organized.

- $\mathrm{FR}_{3}$ : Move the motorcycle around the shop.

- $\mathrm{FR}_{4}$ : Stays stable when parts are removed.

- $\mathrm{FR}_{5}$ : Take maximum $1 \mathrm{~m}^{2}$ of floor space when not in use.

\subsection{Design parameters}

Design parameters (DPs) are the elements of the design solution in the physical domain that are chosen to satisfy the specified FRs [3]. As in the previous domains, the DPs are decomposed from a top-level DP0. [4] suggests that DPs should start with a noun or quantity and be something that can be compared or quantified.

- $\mathrm{DP}_{0}$ : Scissor lift mechanism with extended legs, tool/part tray/magnet and oil catch pan

- $\mathrm{DP}_{1}$ : Hydraulic cylinder and a scissor mechanism

- $\mathrm{DP}_{2}$ : Tool tray/magnet and a oil catch pan

- $\mathrm{DP}_{3}$ : Wheels on undercarriage

- $\mathrm{DP}_{4}$ : Spring-loaded hold-down mechanism

$-\mathrm{DP}_{5}$ : Folding undercarriage/extension legs with wheels

\subsection{Design matrix}

The design matrix (see Equation 2) is then constructed to see how the FR's are related i.e. how one FR affects the other. The goal here is to have a diagonal design matrix, which means no FR affects another FR, which we were able to accomplish. We are therefore not worried about the order of elements in the design and are not afraid of disabling other parts by changing the size and shape of each individual part.

$$
\left\{\begin{array}{l}
\mathrm{FR}_{1} \\
\mathrm{FR}_{2} \\
\mathrm{FR}_{3} \\
\mathrm{FR}_{4} \\
\mathrm{FR}_{5}
\end{array}\right\}=\left[\begin{array}{ccccc}
X & 0 & 0 & 0 & 0 \\
0 & X & 0 & 0 & 0 \\
0 & 0 & X & 0 & 0 \\
0 & 0 & 0 & X & 0 \\
0 & 0 & 0 & 0 & x
\end{array}\right]\left\{\begin{array}{l}
\mathrm{DP}_{1} \\
\mathrm{DP}_{2} \\
\mathrm{DP}_{3} \\
\mathrm{DP}_{4} \\
\mathrm{DP}_{5}
\end{array}\right\}
$$

\subsection{Our Design}

All the group members are motorcycle owners or former motorcycle owners, so all had an opinion what would make the perfect lift. Our assumption was that the most common service operations were on tires and the suspension which requires that the front and/or back part of the motorcycle be disassembled. That creates a large moment on the base of the lift which must be taken into account. In our concept, the gap between the front and back leg is more than the width of the lift to account for the moment. A spring-loaded mechanism is used to ensure the bike is fixtured to the lift and doesn't tip over.

They are an oil catch pan underneath the top plate and magnets on the side to hold the tools in one place while working on the motorcycle. The weight of motorcycles can vary between different brands and sizes so it can be very hard to lift the motorcycle up on the stand. Therefore, our lift slides right under the motorcycle when in its lowest position, eliminating the need to lift the motorcycle up on the lift. A lift without wheels can't be moved around very easily. If the wheels are permanently engaged the risk of accidentally moving the lift while applying force to the motorcycle increases rapidly. Our lift would have wheels that can be engaged and disengaged by simply locking or unlocking them. A lift with this feature set could not be found on the Internet.

Our lift can be seen in early stage of design Figure 3 and in last stage of design in Figure 4.

\subsection{Description of the design}

One of our goals was to be able to slide the lift under the motorcycle. To accomplish this, the oil catch pan had to be mounted on the lift after the lift was raised to highest position (Figure 4). The oil catch pan (Figure 5) is mounted with hooks that are hooked to the oil catch pan: the holes that the hooks go into can be seen in Figure 5. The average amount of engine oil in a motorcycle is $1.5 \mathrm{~L}$. The volume of the oil catch pan is $4 \mathrm{~L}$ so the oil catch pan wouldn't be full when the engine would be drained, making it easier to remove the oil catch pan without spilling the oil. A $3 / 8$ " threaded hole is on the bottom of the oil catch pan for draining with a mini valve. 


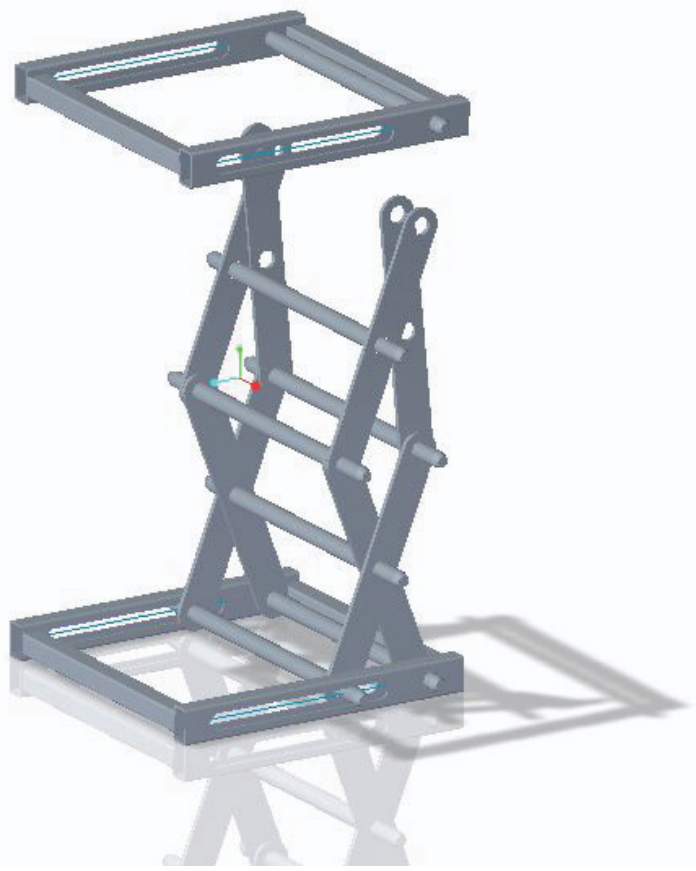

Fig. 3. Lift in first stage of design

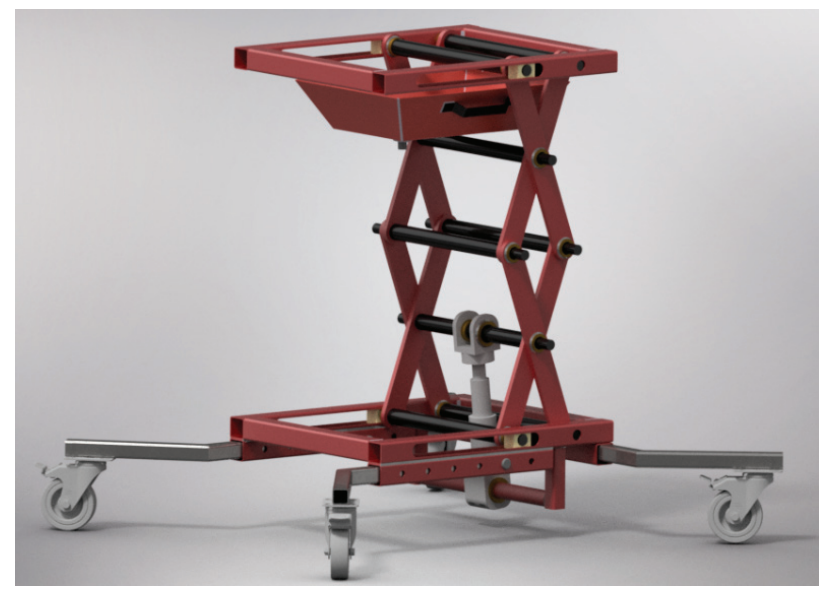

Fig. 4. Lift in highest position with oil catch pan

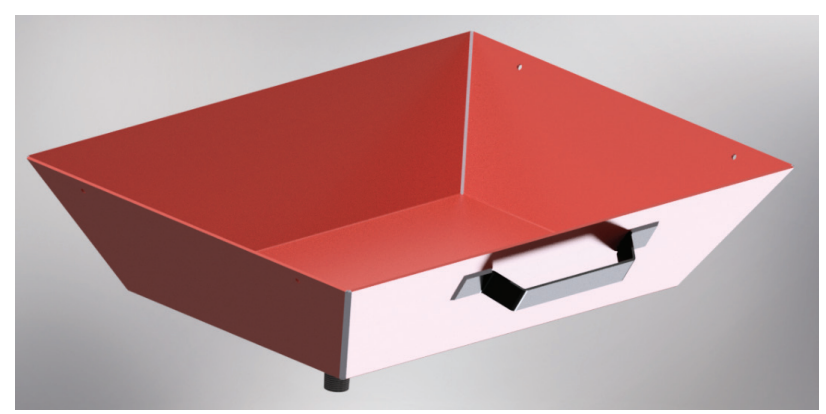

Fig. 5. Oil catch pan

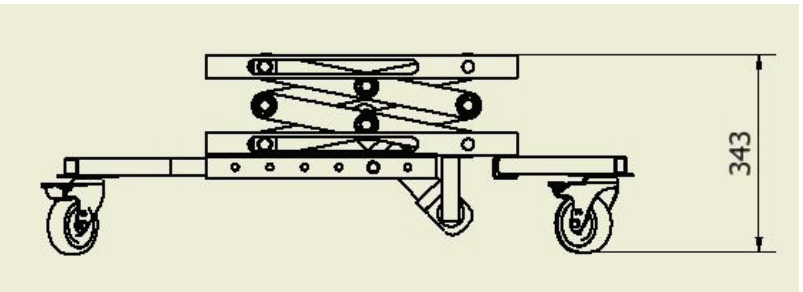

Fig. 6. Height of the lift in lowest position, dimensions in $\mathrm{mm}$

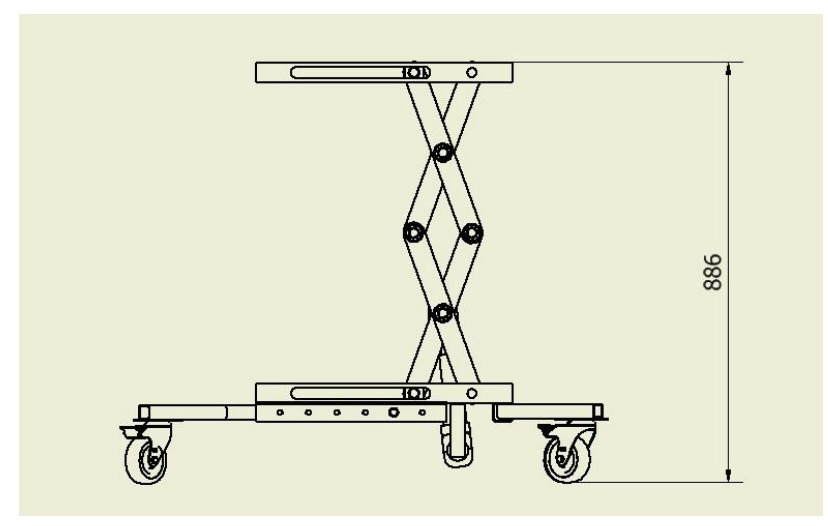

Fig. 7. Height of the lift in highest position, dimensions in $\mathrm{mm}$

When the lift is in lowest position the height is $343 \mathrm{~mm}$, seen in Figure 6, and in the highest position, the height is $886 \mathrm{~mm}$, as can be seen in Figure 7.

One of our goals was to have a securing mechanism that would engage automatically when the motorcycle was being worked on. To accomplish this, the hold-down spring mechanism connects the motorcycle foot-pegs to the highest scissor axle. When the lift is raised to highest position, it applies tension on the spring mechanism because the length has increased. You can observe the difference in length from the top plate of lift and highest scissor axle in Figure 6 and Figure 7.

\subsection{Materials}

The scissor lift is made primarily of regular steel profiles. Other parts needed to build motorcycle lift are two springs to hold motorcycle stuck on the lift, four strong swivel wheels so it can be easily moved around, oil catch pan made from sheet metal to make oil change easier, a magnetic bar for tools, a hydraulic cylinder, fasteners, and paint.

The total cost of the building the prototype (not including the hydraulic cylinder) is approximately 25000 ISK. The first idea was to use hydraulic cylinder found from StarHydraulics [5] which costs 299 USD and shipping cost would be about 80 USD. Price and shipping including the VAT $(24 \%)$ the total cost of the hydraulic cylinder would be around 53570 ISK. Due to the high price and lead time, we found more practical to use standard hydraulic bottle jack instead of the hydraulic cylinder they are listed 


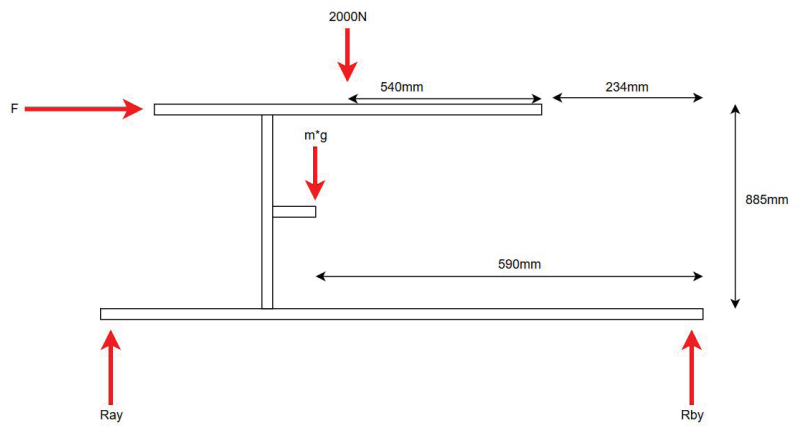

Fig. 8. Force diagram for our lift

for around \$20 on eBay and are easily available in many places. The total material cost would end up to be around 30000 ISK.

\section{Analysis}

\subsection{Comparison}

The stability of the lift is a major aspect of the design. An unstable lift is both dangerous to the user and the stability affects the usability of the mechanism (Section 3.3). To calculate the stability of the lift, we looked at the maximum force that might be applied horizontally on the motorcycle without the lift tilting over. We set up a free body diagram as seen in Figure 8, and use Equation 3 to calculate moment around point $\mathrm{B}$. We assume the lift to be simply supported and the reaction force at point $\mathrm{A}$ to be equal to zero (reaction force at point $\mathrm{A}$ becomes a negative when the moment due to the horizontal force becomes larger than the moments from the masses of the lift and the motorcycle). We look only at the case when the lift is almost tilting over (sign of the reaction force is flipping) and we assume the force due to the mass of the motorcycle to be $2000 \mathrm{~N}(200 \mathrm{~kg})$. Maximum allowable force (without the lift tilting over), when the lift is in the position of maximum height and the wheel legs are in the shortest mode, is $F=1492 \mathrm{~N}$. The maximum force when legs are fully extended is $F=2098 \mathrm{~N}$.

However, using the same method to calculate theoretical maximum vertical force before tipping over will give us a statically indeterminate problem because the force never reaches out of the bottom frame (no negative torque), and therefore the vertical stability ratio is infinite. To compare this to other designs we calculated the same maximum horizontal force for another lift on the market. The result of this analysis is that the maximum force before tilting over was $F=1118 \mathrm{~N}$, which shows the huge advantage of our design because the maximum lifting height is significantly lower in the design of this lift.

$$
\tau=F l
$$

Equation 3 is used to calculate the moment, where $\tau$ is moment in $\mathrm{N} \mathrm{m}, F$ is force in $\mathrm{N}$ and $l$ is distance from the force applied to the point where the moment is calculated in $\mathrm{m}$.
Table 1. Loads and boundary conditions.

\begin{tabular}{ll}
\hline Placement & Load [N] / boundary cond. \\
\hline Top & $4 \times 500$ \\
Center of mass & Gravity \\
Outside edges of legs & Fixed \\
\hline
\end{tabular}

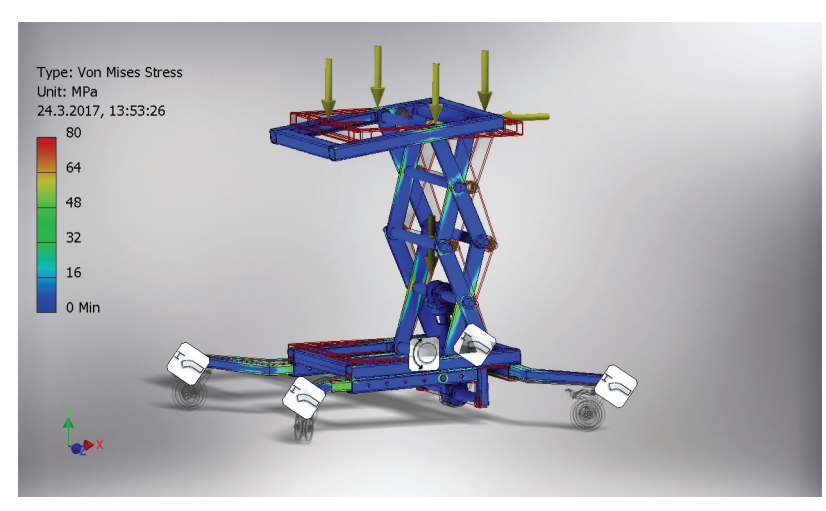

Fig. 9. Result of stress analysis.

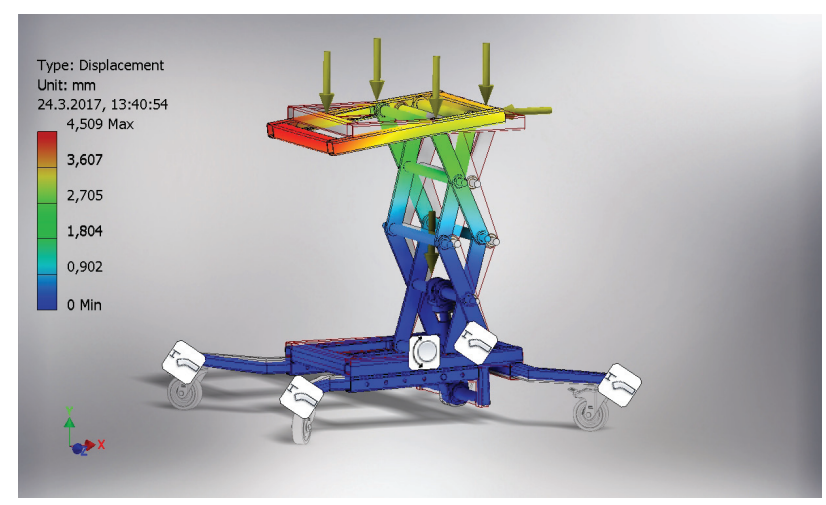

Fig. 10. Displacement under maximum load.

\subsection{Structural analysis}

It is important to analyze if the lift can withstand the load required by the Functional Requirements (Section 3.3). The loads and the boundary conditions for the structural analysis can be seen in Table 1 .

As seen in Figures 9, 10, and 11, the stresses never exceed the yield strength of the materials used (the safety factor is acceptable) and the maximum displacement under the maximum load is about $4.5 \mathrm{~mm}$.

\section{Conclusion}

This motorcycle lift will help make motorcycle maintenance easier than ever. The tool tray will help people using the lift to remember where they put their tools and bolts since there is a convenient place to store them on the motorcycle lift, all in one place. The lift will be lower than a regular motorcycle lift which avoids having to lift the motorcycle on top of the lift first. It will lift the motorcycle high enough to reach an ergonomic working height 


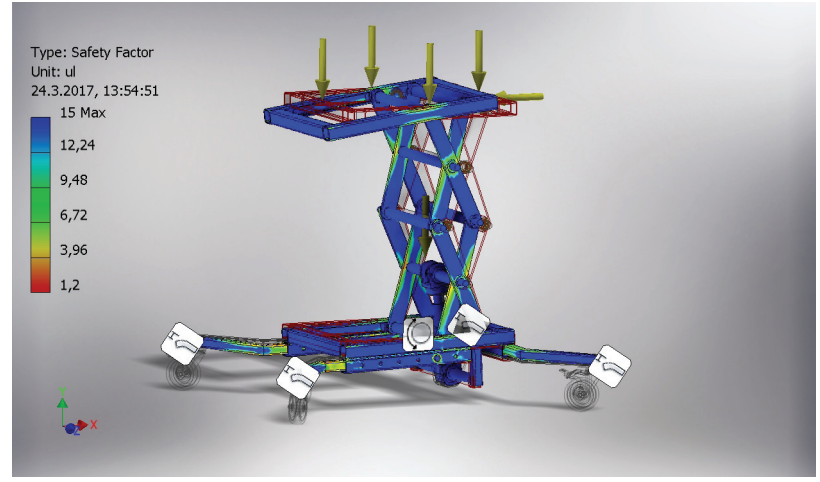

Fig. 11. Safety factor of the design under maximum load.

so the user can stand upright while working on the bike. The design has wheels on the bottom, which can be engaged and disengaged at will, to make it easier to move the lift around a garage even if the motorcycle is on the lift in full working height. The lift has a robust fixturing mechanism to hold the bike on the lift and prevent the bike from falling off the lift. The design is compact, occupying so little space that it can placed almost anywhere while storing it between maintenance sessions. All functional requirements of the design are fully satisfied, as listed:

- $\mathrm{FR}_{1}$ is satisfied with a height of $886 \mathrm{~mm}$ when in top position: a comfortable working height while standing up.

- $\mathrm{FR}_{2}$ is satisfied with an attached magnetic bar so the user can keep all his tools in one place. The oil catch pan will keep the lift and working area clean from oil spilling when changing engine oil.

- $\mathrm{FR}_{3}$ is satisfied with locking swivel wheels so it is easy to roll it around the garage floor.
- $\mathrm{FR}_{4}$ is satisfied because the self-locking spring fixture keeps the bike from falling off the lift when parts are removed.

- $\mathrm{FR}_{5}$ is satisfied with extension legs to add stability for the swivel wheels so the lift only takes $0.76 \mathrm{~m}^{2}$ of space when fully compact.

We can say that our motorcycle lift design is a successful design and all requirements are satisfied. Axiomatic Design greatly helped make our design process systematic and sure that we had clear goals for the project and guidance to how they should integrated.

\section{References}

[1] Discount Ramp, Black widow steel motorcycle jack - 1,100 lb capacity, accessed 2017-03-22, http: //www . discountramps . com/motorcyclescissor-jack/p/BW-1604/

[2] Harbor Freight Tools, Pittsburgh motorcycle 1000 lb. capacity motorcycle lift, accessed 2017-03-22, http: //www . harborfreight . com/automotivemotorcycle/lifts-stands/1000-1bcapacity-motorcycle-lift-69904.html

[3] N.P. Suh, The Principles Of Design (Oxford University Press, Inc., 1990)

[4] D.S. Cochran, J.T. Foley, Z. Bi, Use of the Manufacturing System Design Decomposition for Comparative Analysis and Effective Design of Production Systems, International Journal of Production Research p. 24 (2016)

[5] StarHydraulics, Item hf500-7in (stock), 1 ton (t) foot - operated hydraulic jacks (2017), accessed 201703-22, http://hydraulic-pumps.starhyd.com/ item/lift-jacks/1-ton-t-foot-operatedhydraulic-jacks/hf500-7in-stock 